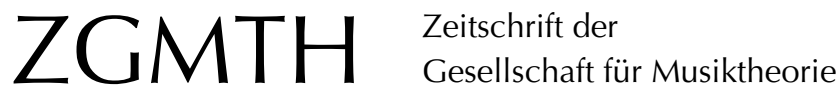

Egermann, Hauke (2013): Zum Zusammenhang von musikalischer Erwartung und Emotion. ZGMTH 10/2, 327-342. https://doi.org/10.31751/725

(C) 2013 Hauke Egermann

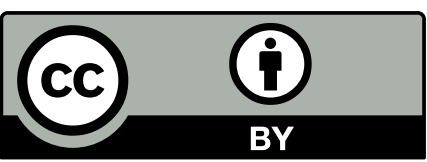

Dieser Text erscheint im Open Access und ist lizenziert unter einer Creative Commons Namensnennung 4.0 International Lizenz.

This is an open access article licensed under a

Creative Commons Attribution 4.0 International License.

veröffentlicht / first published: 17/10/2014

zuletzt geändert / last updated: 19/02/2016 


\title{
Zum Zusammenhang von musikalischer Erwartung und Emotion
}

\author{
Hauke Egermann
}

\begin{abstract}
Im Zentrum dieses Beitrags steht der Zusammenhang zwischen musikalischer Struktur, Hörererwartung und emotionalen Reaktionen. Dazu werden einleitend verschiedene konzeptionelle Arbeiten zum Verhältnis von Musik und Emotionen vorgestellt. Daraufhin wird erläutert, wie Hörererwartungen durch statistisches Lernen ausgebildet werden können. Schließlich werden einige theoretische und empirische Arbeiten präsentiert, die Zusammenhänge zwischen musikalischen Erwartungen und Emotionen erklären und überprüfen. Neben anderen Modellen wird hier das ITPRA-Model von David Huron ausführlich diskutiert. Im Anschluss wird auch eine eigene Studie vorgestellt, die den postulierten Zusammenhang zwischen statischen Eigenschaften musikalischer Strukturen, subjektivem Erwartungserleben und emotionalen Reaktionen bestätigt. Dabei konnte beobachtet werden, dass melodische Segmente mit hohem Informationsgehalt als unerwartet und emotionsinduzierend erlebt werden (verglichen mit Segmenten mit geringem Informationsgehalt).
\end{abstract}

This article focusses on the relationship between musical structures, listener expectations, and emotional reactions. As a starting point, different scientific concepts linking music and emotion are reviewed, followed by an explanation of how listener expectations may be developed through statistical learning. Finally, several further theoretical and empirical approaches explaining the connections between musical expectations and emotions are introduced, including David Huron's ITPRA-Model. A study conducted by the author confirmed the hypothesized relationship between statistical properties of musical structures and emotional reactions. This study demonstrated that melodic segments with high information content are experienced as unexpected and induce emotion (compared to segments with low information content).

Bereits Arthur Dart Bissell ${ }^{1}$ theoretisierte vor fast 100 Jahren über die emotionale Wirkung von erfüllten und unerfüllten musikalischen Erwartungen. Leonard B. Meyer griff knapp 50 Jahre später diese Ideen auf und entwickelte eine Theorie, die postuliert, dass musikalische Emotionen beim Verletzen ${ }^{2}$ von Erwartungen erzeugt werden. ${ }^{3}$ Diese Arbeit sti-

1 Bissell verfasste 1921 eine Dissertation zum Zusammenhang zwischen Musikstrukturen und Erwartungen (Bissell 1921), die vermutlich aufgrund ihres sehr deskriptiven Charakters in der Folge wenig Beachtung fand. Trotz der Tatsache, dass hier kaum theoretisch fundierte Erklärungen für die beschriebenen Zusammenhänge geliefert wurden, ist Bissell meinem Kenntnisstand nach einer der ersten Autoren, der die Bedeutung von Erwartungen für das Musikerleben so ausführlich beschrieb.

2 In Anlehnung an den im anglo-amerikanischen Sprachraum in diesem Kontext oft verwendeten Ausdruck »violation of expectation « wird im Folgenden das Enttäuschen bzw. das Nicht-Entsprechen von Erwartungen als »Verletzen« von Erwartungen bezeichnet. 
mulierte wiederum eine Reihe theoretischer und empirischer Studien, die diesen Zusammenhang überprüften und unterschiedliche Erklärungsmodelle lieferten. Der vorliegende Artikel wird diese Arbeiten überblicksartig vorstellen, ihre Implikationen diskutieren und Forschungsdesiderate aufzeigen. Dabei soll insbesondere ein eigenes empirisches Forschungsprojekt zur Sprache kommen, welches einige der postulierten Zusammenhänge überprüfen konnte. Bevor allerdings der Zusammenhang zwischen Hörererwartung und Emotion genauer betrachtet wird, sollen zunächst einige theoretische Grundprobleme zum Verhältnis von Musik und Emotionen skizziert werden.

\section{Emotionen}

Was sind Emotionen eigentlich? Beverley Fehr und James A. Russell brachten die Problematik hinter dieser Frage auf den Punkt, indem sie als Ergebnis einer Befragungsstudie unter Studierenden konstatierten: »everyone knows what an emotion is, until asked to give a definition «. ${ }^{4}$ Dieses Zitat bringt die Problematik zum Ausdruck, dass zwar jeder ein intuitives Verständnis des Begriffs Emotion hat, insbesondere hinsichtlich der mit dem Begriff assoziierten subjektiven Gefühlsqualitäten, jedoch aufgrund der Komplexität des Phänomens Emotion Schwierigkeiten hat, eine eindeutige Definition zu formulieren. In der Emotionspsychologie existiert dennoch eine Vielzahl an Emotionsmodellen, die meistens hinsichtlich folgender Punkte übereinstimmen: Menschliche Emotionen werden verstanden als durch die Evolution hervorgebrachte Verhaltenssteuerungsmechanismen, die eine flexible Adaption an sich verändernde Umweltverhältnisse ermöglichen. ${ }^{5}$ Einigkeit besteht weiterhin darin, dass diese Mechanismen durch eine synchrone Aktivierung verschiedener Emotionskomponenten realisiert werden, die eine optimale Verhaltensreaktion erlauben. Als Komponenten dieser emotionalen Episoden werden hier meist beschrieben:

a) die kognitive Bewertung eines intern oder extern auftretenden Ereignisses,

b) eine angemessene Verhaltensreaktion (z. B. »flight or fight«), die

c) durch gleichzeitige physiologische Erregung unterstützt wird (z. B. erhöhter Herzschlag oder Hautleitwert),

d) das Kommunizieren/Ausdrücken der Emotion (z. B. Gesichtsausdruck oder Körperhaltung), sowie schließlich

e) das dabei subjektiv empfundene Gefühl. ${ }^{6}$

Damit wird deutlich, dass diesem Verständnis zufolge Emotionen nicht mit Gefühlen gleichzusetzen sind, denn Letztere machen nur einen Teil einer emotionalen Episode aus. Die Bewertungskomponente wird häufig als mehr oder weniger bewusster kognitiver Evaluationsprozess beschrieben, der die eintretenden Ereignisse hinsichtlich ver-

3 Meyer 1956.

4 Fehr/Russell 1984, 464.

5 Sokolowski 2002, 354.

6 Scherer 2005. 
schiedener Dimensionen wie etwa Zielkongruenz, Überwindbarkeit, Vorhersagbarkeit, oder Normkomptabilität einordnet. ${ }^{7}$ Diesem kognitivistischen Verständnis nach entstehen Emotionen als Resultat dieses Bewertungsprozesses, welcher in der einschlägigen Literatur mit dem Begriff Appraisal bezeichnet wird. So kann beispielsweise Wut aus einem überwindbar scheinenden zielhinderlichen Ereignis resultieren, und Trauer, wenn ein zielhinderliches Ereignis nicht überwunden werden kann. Dabei wird oft argumentiert, dass die bei der Emotionsentstehung bewerteten Ereignisse ursprünglich eine überlebensrelevante Bedeutung für den reagierenden Organismus gehabt hätten. So wird ein bedrohliches Tier, das bereits dem Urmenschen in der Wildnis begegnete, zum Emotionsauslöser, sodass noch heute bei einer Begegnung mit einem solchen Tier eine emotionale Reaktionskaskade aktiviert wird, die eine angepasste und überlebensförderliche Reaktion wie Angriff oder Flucht ermöglicht.

Wenn wir uns nun wieder auf die Ausgangsfragestellung nach dem Zusammenhang zwischen musikalischer Erwartung und Emotion besinnen, wird der eine oder andere Leser sich vielleicht fragen, ob dieser Ansatz wirklich in der Lage ist, die Entstehung speziell von musikinduzierten Emotionen zu erklären, da schwer ersichtlich ist, inwiefern das Hören von Musik mit dem Erreichen oder Nicht-Erreichen von überlebenswichtigen Zielen in Zusammenhang steht. So gibt es in der musikpsychologischen und -philosophischen Literatur Positionen, die grundsätzlich verneinen, dass Musikhören - genauso wie andere alltägliche Ereignisse - echte Emotionen zu induzieren vermag. ${ }^{8}$ So vermutet etwa Vladimir Konečni, ${ }^{9}$ dass echte Emotionen beim Musikhören nur induziert werden, wenn diese durch außermusikalische Assoziationen vermittelt sind (wie z.B. eine mit dem Musikstück verbundene Erinnerung an ein bestimmtes emotional besetztes Ereignis). Andere Forscher wiederum argumentieren, dass musikinduzierte Emotionen einer Art Sonderkategorie zuzurechnen sind, nämlich der Kategorie der ästhetischen Emotionen`, weil nicht alle Reaktionskomponenten beim Musikhören durch das Ausbleiben von verhaltensmotivierenden Reaktionstendenzen (wie Annäherung oder Vermeidung) aktiviert werden. ${ }^{10}$ So kann ein Musikstück z. B. im Hörer ein angstähnliches Gefühl induzieren, ohne dass eine Vermeidungstendenz zu beobachten wäre. Insbesondere in den letzten Jahren wurden einige alternative theoretische Erklärungen zur musikbezogenen Emotionsinduktion entwickelt, die im folgenden Abschnitt vorgestellt werden sollen.

\section{Musik und Emotion}

Vor dem Hintergrund von Unzulänglichkeiten des Modells der kognitiven Emotionsentstehung fassen Patrik N. Juslin und Daniel Västfjäll ${ }^{11}$ eine Reihe von weiteren Emotionsentstehungsmechanismen zusammen und beschreiben auch einige empirischen Studien, die nachweisen konnten, dass bei Musikhörern eine Vielzahl an affektiven Phänomenen

7 Scherer 1999, 639.

8 Kivy 1990.

9 Konečni 2008.

10 Scherer 2004, 241-244.

11 Juslin/Västfjäll 2008. 
induziert wurden. Letztere waren in ihrem Ablauf der im vorherigen Abschnitt idealisierten emotionalen Reaktionskette sehr ähnlich. So konnte beispielsweise die Arbeitsgruppe um Robert Zatorre ${ }^{12}$ während starker musikinduzierter Emotionen Aktivierungen tiefer subkortikaler Gehirnstrukturen nachweisen, die normalerweise nur mit evolutionsrelevanten Stimulationen wie Essen, Fortpflanzung oder Drogenkonsum in Verbindung gebracht wurden. Auch ließen sich ähnliche musikalische `Peakı-Momente mit synchronisierten Emotionskomponentenaktivierungen beobachten: Egermann et al. ${ }^{13}$ zeigten z. B., dass Veränderungen der subjektiven emotionalen Intensität mit Aktivierungen des sympathischen Nervensystems einhergingen. Somit kann die Frage nach der Existenz von sechten s musikinduzierten Emotionen bejaht werden, jedoch stellt sich weiterhin die Frage, welche theoretischen Entstehungsmodelle diese Zusammenhänge erklären könnten. Juslin und Västfjäll ${ }^{14}$ führen hierzu einige weitere Mechanismen an, die die eingangs erläuterten kognitiven Bewertungsprozesse komplementieren:

'Brain stem reflexes` (Hirnstamm-Reaktionen) beschreiben emotionale Reaktionen, die aufgrund von basalen akustischen Eigenschaften wie Lautheit oder sensorischer Dissonanz durch Reaktionsmechanismen im Hirnstamm erzeugt werden und damit wichtige und dringliche Veränderungen des akustischen Umfeldes signalisieren. `Visual imagery، (visuelle Imagination) bezeichnet einen Prozess, bei dem durch Musik hervorgerufene visuelle Eindrücke (wie zum Beispiel emotional stimulierende Landschaftsbilder) Emotionen induzieren. Zwei weitere Mechanismen basieren auf der Verknüpfung von Musik mit anderen emotionalen Gedächtnisinhalten: `Evaluative conditioning` (evaluatives Konditionieren) liegt vor, wenn ein musikalischer Stimulus wiederholt mit einem unkonditionierten emotionalen Stimulus gepaart präsentiert wird (z. B. dem Treffen eines guten Freundes beim Musikhören). 'Episodic memory` (episodisches Gedächtnis) induziert Emotionen, wenn im Voraus emotional konnotierte persönliche Ereignisse von ursprünglich neutraler Musik begleitet werden (wie z. B. Musik im Kontext einer Beerdigung oder Trauung). Bei semotional contagion` (emotionaler Ansteckung) führen der Musik zugeschriebene emotionale Ausdrucksgesten durch mentales Imitieren durch den Hörer zu vergleichbaren Emotionen. ${ }^{15}$ Schließlich beschreiben Juslin und Västfjäll noch, wie das Erfüllen oder Verletzten von Erwartungen Emotionen erzeugen kann. Diesen Mechanismus bezeichnen sie als ımusical expectancy

Dass hier nicht weniger als sieben verschiedene Mechanismen genannt werden, die vermutlich gleichzeitig und unabhängig voneinander Reaktionen während der Musikrezeption erzeugen, macht deutlich, was für eine hohe Komplexität das Entstehen von musikinduzierten Emotionen aufweist. So können den Autoren nach die unterschiedlichen Mechanismen auch simultan unterschiedliche affektive Qualitäten erzeugen, wodurch sich die Reichhaltigkeit des emotionalen Erlebens von Musik erklären lässt. Es bleibt jedoch anzumerken, dass bisher lediglich ein Teil dieser Mechanismen empirisch nachgewiesen werden konnte.

12 Blood/Zatorre 2001 und Salimpoor/Benovoy/Larcher/Dagher/Zatorre 2011.

$13 \mathrm{Vgl}$. Egermann/Sutherland/Grewe/Nagel/Kopiez/Altenmüller 2011.

14 Juslin/Västfjall 2008.

15 Vgl. auch Egermann/McAdams 2013. 
Insbesondere die Rolle von musikalischen Erwartungsprozessen in der Emotionsentstehung ist bislang nur teilweise experimentell belegt worden. Diesem Mechanismus kommt vor dem Hintergrund der oben erwähnten konzeptionellen Probleme allerdings eine besondere Bedeutung bei der Erklärung von musikinduzierten Emotionen zu. Im Gegensatz zu den anderen sechs Mechanismen steht er direkt mit der komponierten und interpretierten Struktur von Musik in Verbindung. Gelänge ein experimenteller Nachweis dieses Mechanismus, ließe sich damit auch aufzeigen, dass a) musikalische Strukturen aus sich selbst heraus, d. h. ohne außermusikalische Referenzen, in der Lage sind, emotionale Reaktionen beim Hörer zu evozieren ${ }^{16}$ und b) eine expektanzorientierte Analyse musikalischer Strukturen bis zu einem gewissen Grad in der Lage ist, Hörerleben vorherzusagen. ${ }^{17}$ Der zuletzt genannte Punkt ist vermutlich die Ursache dafür, dass insbesondere in der nordamerikanischen kognitivistisch geprägten Musiktheorie nach wie vor ein großes Interesse an diesem Thema besteht.

\section{Musik und Erwartung}

Leonard B. Meyer ${ }^{18}$ erklärt das Entstehen von musikalischen Erwartungen durch das wiederholte Hören syntaktischer Regelmäßigkeiten einer Musikkultur. Eine Fortführung dieser Theorie präsentiert das viel zitierte Buch Sweet Anticipation von David Huron ${ }^{19}$, welcher sich direkt auf Meyers Arbeiten bezieht und auf der Basis aktueller wissenschaftlicher Arbeiten an diese anknüpft. Huron beschreibt vier unterschiedliche Arten von musikalischen Erwartungen, die jeweils von unterschiedlichen (teilweise auditiven) Gedächtnismodulen realisiert werden: `Veridical expectations` (wahrheitsgetreue Erwartungen) entstehen im episodischen Gedächtnis und basieren auf Informationen über die Struktur eines bestimmten bekannten Musikstückes. 'Schematic expectations` (schematische Erwartungen) entstehen durch das alltägliche Hören unterschiedlicher Musikstile und basieren auf verallgemeinerten Übergangswahrscheinlichkeiten musikalischer Ereignisse innerhalb dieser Musikstile. ${ }^{20}$ Sie werden nach Huron durch das semantische Gedächtnis realisiert. `Dynamic expectations` (dynamische Erwartungen) basieren auf Erwartungen innerhalb eines Musikstückes, welche im Kurzzeitgedächtnis des Hörers in Echtzeit generiert werden. Schließlich beschreibt Huron noch mit sconscious expectations` bewusste Erwartungen während des Hörens von Musik. So kann z. B. das mehrfache Wiederholen eines Motivs innerhalb einer Komposition eine dynamische Erwartung aufbauen, die nur Gültigkeit für das momentane Hören dieses Stückes hat. Wird das Stück mehrfach gehört, bilden sich wahrheitsgetreue Erwartungen, die z. B. beim Spiel einer falschen Note durch einen Interpreten verletzt werden können. Wenn eine Vielzahl an verschiedenen Stücken eines Musikstils wiederholt gehört wird, bilden sich schematische Erwartungen aus, die z. B. durch besonders häufige Akkordfolgen innerhalb eines

16 Cochrane 2010, 984.

17 Margulis 2005, 664.

18 Meyer 1956.

19 Huron 2006.

20 Zur Unterscheidung zwischen ıveridicalı und sschematic expectations « siehe auch Bharucha/Todd 1989. 
Stiles entstehen. Erklingt ein anderes als das im Programm eines Konzerts abgedruckte Stück, können nach Huron bewusste Erwartungen verletzt werden. Ein und dasselbe Ereignis innerhalb eines bekannten Musikstückes kann nicht nur wahrheitsgetreue Erwartungen erfüllen, sondern zugleich auch - wenn es stilistisch ungewöhnlich ist - schematische Erwartungen verletzen. Ähnliches kann bei der Variation eines bereits mehrfach wiederholten Motivs passieren; hier ist es jedoch dann eine dynamische Erwartung, die durchkreuzt wird. Damit wird klar, dass dieser Theorie nach das Hören eines Musikstückes unterschiedlichste, voneinander unabhängige Erwartungserfahrungen mit sich bringen kann, die mehr oder weniger bewusst ablaufen.

Hurons Beschreibungen basieren nur zum Teil auf empirischen Beobachtungen, eine Überprüfung dieser feinen Differenzierung musikalischer Erwartungen ist meines Wissens bisher noch nicht geschehen. Viele Studien befassen sich stattdessen mit Erwartungsarten, die durch unterschiedliche musikalische Parameter wie Melodie, Harmonie und Rhythmus/Metrik verursacht werden. So wurde beispielsweise gezeigt, dass rhythmische Periodizitäten präattentive hierarchische Erwartungen von Metrum und Takt erzeugen. ${ }^{21}$ Harmonische Erwartungen wurden überprüft, indem Reaktionen auf Akkorde mit unterschiedlichen kontextuellen harmonischen Verwandtschaftsgraden verglichen wurden. Hierbei wurde beobachtet, dass sich die Antworten der Versuchspersonen verzögerten, wenn weiter entfernte mit näher liegenden Akkorden verglichen wurden (die damit vermutlich weniger erwartet waren). ${ }^{22}$ Hierbei wurde der Akkordverwandtschaftsgrad durch die Anzahl an gleichen Grundtönen quantifiziert. Des Weiteren führten diese Akkorde zu verringerten subjektiven Vollständigkeits- und Erwartungsbewertungen, ${ }^{23}$ welche auch von entsprechenden ereigniskorrelierten Gehirnaktivitätspotentialen im Elektroenzephalogramm (EEG) begleitet wurden. ${ }^{24}$ Bezüglich der mit Melodien im Zusammenhang stehenden Erwartungen sind verschiedene theoretische Modelle vorgeschlagen worden, ${ }^{25}$ welche jedoch bis dato nur teilweise empirisch überprüft wurden. Eugene Narmour stellte mit seinem implication-realization model eine der einflussreichsten Theorien zur Vorhersage von melodischen Erwartungen vor. ${ }^{26}$ Er schlug mehrere melodische Prinzipien vor, die erwartbare Fortschreitungen von Intervallen beschreiben. Einige dieser (gestalttheoretisch inspirierten) Prinzipien konnten in experimentellen Hörversuchen bestätigt werden (z. B. die Erwartung von Nähe), andere wiederum nicht. ${ }^{27}$ Hieran kann das Potential einer produktiven Wechselwirkung zwischen empirischer Forschung und Musiktheorie veranschaulicht werden: Empirische Methoden und Theoriemodelle können sich gegenseitig zu Präzisierung und Verfeinerung anregen.

Im Unterschied zu Meyer verstand Narmour einige dieser melodischen Organisationsprinzipien als universelle und angeborene Prozesse. ${ }^{28}$ Andere Theorien schlagen je-

21 Ladinig/Honing/Háden/Winkler 2009 und Zanto 2006.

22 Bharucha/Stoeckig 1986.

23 Bigand/Pineau 1997 und Schmuckler/Boltz 1994.

24 Janata 1995 und Carrion/Bly 2008.

25 Vgl. z. B. Narmour 1990 und 1992, Larson 2004, Margulis 2005 sowie Ockelford 2006.

26 Narmour 1990 und 1992.

27 Vgl. z. B. Cuddy/Lunney 1995, Schellenberg 1996 und Thompson/Stainton 1998. 
doch (wie Huron) vor, dass melodische Erwartungen vollständig durch das wiederholte und passive Hören von syntaktischen Relationen der musikalischen Struktur gebildet werden (als auditives statistisches Lernen). ${ }^{29}$ Ferner konnte gezeigt werden, dass computerbasierte Stimulationen dieses statistischen Lernprozesses gute Vorhersagen des subjektiv bewerteten Erwartungsgrads melodischer Ereignisse leisten. ${ }^{30}$ Es wurde sogar deutlich, dass diese Modelle bessere Erwartungsvorhersagen treffen als Quantifizierungen des Modells von Narmour.

Ein Beispiel eines solchen computerbasierten Modells ist das Information Dynamics Of Music Model (IDyOM), welches in einer Reihe von Arbeiten vorgestellt ${ }^{31}$ und empirischen Überprüfungen unterzogen wurde. ${ }^{32}$ Das Modell lernt sunüberwacht durch die Analyse von sequenziellen Abhängigkeiten zwischen Tönen in Melodien. Dabei generiert das Modell zu jedem Zeitpunkt einer Melodie eine Wahrscheinlichkeitsverteilung einer bestimmten Eigenschaft des nächsten Tons (z. B. Tonhöhe oder Einsatzzeitpunkt). Diese Wahrscheinlichkeitsverteilung reflektiert dabei die Erfahrung des Modells und erlaubt somit eine Quantifizierung des Erwartungswertes des nächsten Tons. Für jeden erklingenden Ton lässt sich so aus seiner vorhergesagten Eintrittswahrscheinlichkeit sein Informationsgehalt (das ein Maß der Erwartungsdiskrepanz darstellt) berechnen. Das Modell bildet dabei die vermuteten impliziten melodischen Lernprozesse mit zwei kombinierten Teilmodellen ab, welche jeweils einen unterschiedlichen auditiven Gedächtnistyp simulieren sollten: Das Kurzzeitmodell, das als Eingabe nur die zu analysierende Melodie hat, repräsentiert das auditive Kurzzeitgedächtnis, in dem dynamische Erwartungen generiert werden. Das Langzeitmodell wird an einem Korpus von mehreren hundert tonalen Melodien trainiert und soll damit das semantische Gedächtnis (mit schematischen Erwartungen) eines durchschnittlichen westlichen Musikhörers repräsentieren. Die gute Vorhersagekraft dieses statistischen Lernmodells mag als Hinweis auf die Plausibilität der durch das Modell implizierten Annahmen zum Entstehen musikalischer Erwartungen gesehen werden; aber unabhängig davon lässt sich das Modell auch zur automatisierten Musikanalyse verwenden. Auf der einen Seite wurde es bereits angewandt, um musikalische Phrasierungs- bzw. Segmentierungsanalysen anzufertigen (musikalische Phrasengrenzen treten häufig vor Tönen mit hohem Informationsgehalt auf); ${ }^{33}$ auf der anderen Seite wurde das Modell kürzlich angewandt, um Momente erhöhter emotionaler Aktivierung in monophonen Kompositionen zu identifizieren. ${ }^{34}$ Dieses zuletzt genannte Forschungsprojekt soll im Anschluss noch ausführlicher vorgestellt werden. Zunächst bleibt jedoch die Frage zu erörtern, durch welche mentalen Vorgänge Erwartungsprozesse und musikinduzierte Emotionen verknüpft werden.

28 Narmour 1991, 3.

29 Abdallah/Plumbley 2009 und Pearce/Wiggins 2006.

30 Pearce/Wiggins 2006.

31 Pearce 2005 und Pearce/Wiggins 2004.

32 Pearce/Wiggins 2006 und Pearce/Ruiz/Kapasi/Wiggins/Bhattacharya 2010.

33 Pearce/Müllensiefen/Wiggins 2010.

34 Egermann/Pearce/Wiggins/McAdams 2013. 


\section{Musikalische Erwartung und Emotion}

Ganz in der Tradition des amerikanischen Pragmatismus Deweyscher Prägung stehend, erklärt Meyer Emotionen bei verletzten musikalischen Erwartungen wie folgt: »Emotion or affect is aroused when a tendency to respond is arrested or inhibited. " $^{35}$ Musikalische Erwartungen sind demnach für Meyer an Verhaltenstendenzen gebunden, die beim Ausbleiben des Erwarteten unterdrückt werden, woraufhin subjektive Spannung und Emotionalität entsteht.

Eine grundsätzliche Erweiterung dieses recht simplen Wirkungsmodells, das auf das Entstehen von Emotionen beim Verletzen und Enttäuschen von Erwartungen begrenzt ist, liefert Huron. ${ }^{36}$ Seine Theorie beschreibt eine ganze Reihe von emotionalen Phänomenen, die durch musikalische Erwartungen verursacht werden können. Eine prinzipielle Grundannahme von Huron ist, dass unser auditorisches Wahrnehmungssystem kontinuierlich Berechnungen über die Wahrscheinlichkeit zukünftig eintretender Ereignisse durchführt. Dabei werden fünf verschiedene affektive `Response`-Typen unterschieden. Zwei davon treten vor einem erwarteten Ereignis ein: Die simagination response schreibt emotionale Reaktionen, die lediglich aufgrund der bloßen Vorstellung entstehen, dass ein zukünftiges Ereignis eintreten wird. Obwohl diese Art von Reaktion für imaginierte alltägliche Ereignisse recht plausibel erscheint, liefert Huron leider kein Beispiel dafür, wie oder wann eine solche Reaktion beim Erwarten musikalischer Ereignisse auftreten könnte. Der `tension response` widmet Huron jedoch ein gesamtes Kapitel. Sie beschreibt das Entstehen von antizipatorischer Erregung vor dem Eintreten des erwarteten Ereignisses. Die stension response « wird nach Huron verstärkt, wenn der Eintrittszeitpunkt des erwarteten Ereignisses unsicher ist. Des Weiteren beschreibt Huron noch drei Response-Typen, die nach dem vorhergesagten musikalischen Ereignis eintreten: sprediction response und reaction response، sollen zeitgleich auftreten. Die sprediction response beschreibt einen Vorgang, der die Vorhersagegenauigkeit bewertet und entsprechende emotionale Reaktionen generiert: Korrekte Vorhersagen werden durch das Entstehen von positiven Emotionen belohnt und falsche Vorhersagen mit negativen Emotionen bestraft. Die reaction response stellt simultan dazu eine schnelle und weniger akkurate affektive Reaktion auf basale Stimuluseigenschaften dar (wie z. B. Lautheit oder sensorische Dissonanz). Schließlich beschreibt Huron noch die sappraisal response . Hierbei wird eine ausführliche und gegebenenfalls auch bewusste Bewertung des gehörten Ereignisses in Bezug auf dessen Konsequenzen durchgeführt. ${ }^{37}$

Diese fünf unterschiedlichen Response-Arten - >Imagination<, >Tension`, >Prediction`, 'Reaction`, und `Appraisalı (IPTRA) - führen nach Huron in ihrem Zusammenwirken zu verschiedenen affektiven Reaktionen auf musikalische Ereignisse im Zusammenhang mit Erwartungsprozessen. Die dabei entstehenden emotionalen Qualitäten können je-

35 Meyer 1956, 14.

36 Huron 2006.

37 Die Differenzierung zwischen ^reaction response und sappraisal response geschieht in Anlehnung an Joseph LeDoux (1996), welcher in einem neurophysiologischen Modell zwei Wege der Emotionsentstehung beschrieb: einen schnellen und ungenauen sowie einen langsamen und genaueren Weg der Stimulusbewertung (allerdings mit einem Fokus auf die Entstehung von Angst). 
doch unterschiedliche Wertigkeiten haben; so könnte beispielsweise das Verletzen von Erwartungen zunächst eine negative Reaktion verursachen (sprediction response ), die aber darauf in einem Musikrezeptionskontext durch die sappraisal response tiv eingeschätzt wird, da festgestellt werden könnte, dass sich durch das unerwartete musikalische Ereignis keine >Gefahrı ergibt oder man die Musik gerade aufgrund der Erwartungsverletzung insgesamt als angenehm oder interessant bewertet. So kann schließlich eine positive erwartungsinduzierte Emotion entstehen, die Huron als >contrastive valence ${ }^{38}$ beschreibt: Die resultierende Emotion wird durch den Kontrast zwischen ursprünglich negativer Wertigkeit und nachträglich positiver Wertigkeit verstärkt. Obwohl ursprünglich nicht von Huron beachtet, ließe sich hier möglicherweise auch die exitation transfer theory von Dolf Zillmann ${ }^{39}$ auf Musik übertragen: Die durch ein vorausgehendes negatives Ereignis (Erwartungsverletzung) verursachte Erregung wird auf sich anschließende Ereignisse (Bewertung der weiteren Musik) übertragen und führt zu einer Intensivierung sich anschließender Emotionen. Ferner ergeben sich nach Huron positive erwartungsinduzierte Emotionen auch durch das Einbeziehen der Möglichkeit einer positiven sprediction response . Demnach erzeugt das Eintreten von korrekt vorhergesagten Ereignissen positive emotionale Reaktionen. So werden durch wiederholtes Hören desselben Musikstückes ıveridical expectations`, durch stilistische Ähnlichkeit verwendeter Musikstrukturen sschematic expectations und durch Wiederholungen von musikalischer Strukturen innerhalb einer Komposition `dynamic expectations` erfüllt und positive Emotionen induziert. ${ }^{40}$

Obwohl so nicht von Huron dargestellt, könnte die eingangs beschriebene kognitive Bewertungskomponente zur Erklärung der unterschiedlichen Response-Typen verwendet werden. ${ }^{41}$ In den verschiedenen Appraisal-Modellen wird die Vorhersagbarkeit oder Erwartbarkeit des eintretenden Ereignisses häufig als eine von vielen Bewertungsdimensionen genannt. ${ }^{42}$ So könnte ein als unerwartet bewertetes musikalisches Ereignis das subjektive Gefühl von Überraschung induzieren, begleitet von körperlichen Reaktionen, die eine an die Umwelt angepasste Verhaltensantwort unterstützen (wie etwa erhöhte Informationsaufnahme, Unterbrechung von momentan ausgeführten Aktivitäten, auch als sfreezing bezeichnet, oder entsprechende Ausdrucksreaktionen durch Mimik und Gestik). Hurons sprediction response` ähnelt somit einer aus dieser Evaluationsdimension resultierenden Emotion. Des Weiteren werden Emotionen oft als Resultat unterschiedlicher mentaler Verarbeitungsmodule mit unterschiedlichem Grad an Genauigkeit und Geschwindigkeit beschrieben, was der Differenzierung von seaction response versus sappraisal response entspricht. ${ }^{43}$ So wäre zu überlegen, ob sich nicht die sprediction response als ein Teil der reaction response und rappraisal response beschreiben ließe.

38 Huron 2006, 21.

39 Zillmann 1971.

40 Huron, 2006, 239-268.

41 Scherer 1999.

42 Vgl. z. B. Scherer 2004 und Rosemann/Antoniou/Jose 1996.

43 LeDoux 1996. 
Schließlich beziehen die meisten Emotionsmodelle auch häufig die Möglichkeit ein, dass Emotionen aufgrund von imaginierten, also durch von lediglich internen Ereignissen ausgehenden Prozessen generiert werden, was erlaubt, diese Modelle auch zur Erklärung der simagination` und stension responses` zu verwenden. Somit sollte der Beitrag von Hurons IPTRA-Model nicht als Erklärungsmodell zur Entstehung von erwartungsinduzierten Emotionen gewertet werden, sondern eher als Beschreibungsmodell der aus diesem emotionalen Verarbeitungsapparat entstehenden emotionalen Qualitäten.

Schließlich sei noch angemerkt, dass nach Huron musikalische Erwartungsprozesse noch weitere Phänomene im Hörerleben generieren können. So erzeugen statistische Eigenschaften von musikalischen Strukturen Wahrnehmungsqualitäten, die sich von induzierten Emotionen unterscheiden, da vermutlich keine Verhaltensreaktionen ausgelöst werden. So beschreibt Huron beispielsweise, dass diatonische Skalen spezifische Empfindungen wie Schlusswirkung (`closure`), Tendenz (ıtendencys) oder Annehmlichkeit ('pleasure $`$ ) erzeugen. ${ }^{44}$

\section{Empirische Befunde zum Zusammenhang zwischen Erwartung und Emotion}

Die wenigen bisher existierenden experimentellen Befunde zum Zusammenhang zwischen Erwartung und Emotion beschränkten sich zunächst lediglich darauf, emotionale Reaktionen nach dem Eintreten unerwarteter Ereignisse mit erwarteten Ereignissen zu vergleichen. Einen der ersten empirischen Befunde auf diesem Gebiet lieferte eine Studie von John Sloboda, ${ }^{45}$ der Musikexperten befragte, welche musikalischen Strukturen ihrer Erinnerung nach Emotionen induzieren. Dabei wurden insbesondere unerwartete harmonische Wendungen oder plötzliche dynamische oder strukturelle Veränderungen mit dem Erleben von starken emotionalen Reaktionen in Zusammenhang gebracht (welche auch von körperlichen Empfindungen wie einem Schauer oder einer Gänsehaut begleitet wurden). Freilich können diese Einschätzungen nicht als Beleg für die Allgemeingültigkeit dieses Zusammenhangs gewertet werden, da hier schließlich keine musikalischen Stimuli experimentell variiert und Reaktionen gemessen wurden. Die Anlage der Studie bedingt, dass hier eher die abstrahierte und möglicherweise theoriegeleitete Meinung über diese Reaktionen erfasst wurde und weniger das tatsächliche momentane subjektive Erleben der Musikstruktur.

Dagegen führte Mathias Feldmann im Rahmen seiner Dissertation ein experimentelles Forschungsvorhaben durch, bei dem er seinen Probanden drei verschiedene Musikausschnitte mit jeweils vermutlich unterschiedlich starken Erwartungsverletzungen vorspielte und mithilfe einer subjektiven Bewertungsskala veränderte emotionale Reaktionen erfasste. ${ }^{46}$ Das Musikstück, welches mittlere Erwartungsdiskrepanzen induzieren sollte, induzierte jedoch im Gegensatz zu den beiden anderen Stücken mit angenommenen hohen und niedrigen Erwartungsverletzungen die geringste emotionale Aktivierung, was den Autor dazu veranlasste, die Ausgangshypothese (Erwartungsverletzungen

44 Huron 2006, 143.

45 Sloboda 1991.

46 Feldmann 1998. 
bewirken emotionale Intensitätssteigerungen ${ }^{47}$ ) zu verwerfen. Das Ergebnis dieser Studie bleibt jedoch fraglich, da in der Darstellung des Versuchs wesentliche Angaben fehlen und das verwendete Versuchsdesign eine Kovariation von anderen emotionsrelevanten Parametern zwischen den drei verschiedenen Bedingungen von Erwartungsdiskrepanzen nicht ausschließen konnte und somit ein eindeutiger Kausalschluss erschwert wird. ${ }^{48}$

Weitere experimentelle Überprüfungen hinsichtlich des Zusammenhangs zwischen Erwartung und Emotion führte die Arbeitsgruppe um Stefan Koelsch durch. In mehreren Studien konnte hier nachgewiesen werden, dass das Verletzen von harmonischen Erwartungen zur Aktivierung von verschiedenen Emotionskomponenten führt. Dabei wurden originale Schlusswendungen von Bachchorälen oder Klaviersonaten so verändert, dass die letzte erklingende Harmonie entweder der Tonika entsprach (ausgesprochen erwartungskonform) oder dem neapolitanischen Sextakkord (ausgesprochen unerwartet). Beim Vergleich dieser Varianten konnten so für sehr unterwartete Akkorde eine erhöhte subjektive Spannung und Emotionalität, ${ }^{49}$ eine Aktivierung des sympathischen Nervensystems, ${ }^{50}$ eine bilaterale Aktivierung der Amygdala (einer für das Verarbeiten von negativen emotionalen Stimuli zuständigen subkortikalen Gehirnstruktur), die Induktion eines unangenehmen Gefühls ${ }^{51}$ und ereigniskorrelierte EEG-Komponenten der Gehirnaktivität ${ }^{52}$ beobachtet werden. Somit zeigen drei Studien stimulusbezogene Aktivierungen in verschiedenen emotionalen Reaktionskomponenten. Jedoch wurde hier die vermutete Erwartungshaltung der Hörer von den Forschern im Voraus festgesetzt, eine unabhängige Überprüfung der Stimuli hinsichtlich der subjektiven und objektiven Vorhersagbarkeit wurde nicht vorgenommen.

Diese Einschränkungen versuchte ein von mir geleitetes Forschungsprojekt zu umgehen. Das Ziel dieses Projekts war es, die Existenz von erwartungsinduzierten Emotionen in einem ökologisch validen Experimentalansatz zu überprüfen. Dazu wurde ein Konzert organisiert, in dem kontinuierlich verschiedenste Reaktionsmaße von Versuchsteilnehmern aufgezeichnet wurden. Gleichzeitig wurde die präsentierte Musik einer informationstheoretischen Analyse unterzogen, die Vorhersagen darüber generierte, welche musikalischen Ereignisse Erwartungsverletzungen verursachen würden. ${ }^{53}$

47 Feldmann verwendet in Anlehnung an den Emotionspsychologen George Mandler den Begriff >Erwartungsdiskrepanz `Erwartungsverletzung`. Beide Begriffe bezeichnen meinem Verständnis nach jedoch dasselbe Phänomen.

48 So wird zum einen nicht beschrieben, wie die verwendeten drei Musikausschnitte genau erstellt wurden, zum anderen wurde pro Erwartungsverletzungskategorie (klein versus mittel versus groß) nur je ein Musikausschnitt aus drei unterschiedlichen Kompositionen verwendet (weshalb die Faktoren `Komposition

49 Steinbeis/Koelsch/Sloboda 2006.

50 Koelsch/Kilches/Steinbeis/Schelinski 2008.

51 Koelsch/Fritz/Schlaug 2008.

52 Steinbeis/Koelsch/Sloboda 2006.

53 Eine ausführliche Beschreibung der Studie findet sich bei Egermann/Pearce/Wiggins/McAdams 2013. 
Wir organisierten ein Querflötenkonzert und verwendeten ein Messsystem, das am Centre for Interdisciplinary Research in Music Media and Technology (McGill University, Montreal) entwickelt wurde. Dieses System ermöglicht die zeitgleiche Erfassung der Aktivität des autonomen Nervensystems (Herzrate, Atemrate und Hautleitwert), der Aktivität expressiver Gesichtsmuskeln sowie der subjektiven Empfindung der im Voraus rekrutierten 50 Konzertbesucher. Die eine Hälfte der Teilnehmer bewertete dabei über ein digitales Interface in einem zweidimensionalen Raum die Wertigkeit (von unangenehm bis angenehm) und Erregung (von ruhig bis erregt) der eigenen emotionalen Empfindungen während der Musikrezeption. Die zweite Gruppe der Teilnehmer hatte die Aufgabe, die erklingenden musikalischen Ereignisse hinsichtlich ihrer subjektiven Vorhersagbarkeit zu bewerten. Dazu verwendeten sie ein ähnliches Interface, welches jedoch eine eindimensionale Skala von unerwartet (oben) und erwartet (unten) abbildete. Mit ihrem Zeigefinger konnten die Teilnehmer durch Bewegungen auf der Interfaceoberfläche anzeigen, wie emotional bzw. unerwartet sie die Musik erlebten.

Präsentiert wurden sechs Solokompositionen für Querflöte aus verschiedenen Epochen (von Johann Sebastian Bach, Paul Hindemith, Francis Poulenc, Claude Debussy und Edgard Varèse). Der gesamte Notentext der sechs Stücke wurde anschließend mit IDyOM ${ }^{54}$ hinsichtlich der statistischen Vorhersagbarkeit von Tonhöhen analysiert. Nachdem die sechs Stücke einer motivischen Analyse unterzogen wurden, konnten ca. 180 ein- bis zweitaktige Segmente identifiziert werden, für welche die mittlere subjektive `Unerwartetheits (abgeleitet aus den Bewertungen der Teilnehmer) und der mittlere Informationsgehalt (als Ergebnis der Computeranalyse) berechnet wurden. Anschließend konnte beobachtet werden, dass Segmente mit einem besonders hohen Informationsgehalt, verglichen mit denen mit geringem, zu einem signifikanten Anstieg der subjektiven Unerwartetheitsbewertungen führten. Somit wurde nachgewiesen, dass auch in diesem Fall das IDyOM in der Lage war, musikalische Ereignisse zu identifizieren, die von den Versuchsteilnehmern als unerwartet beschrieben wurden.

Alle weiteren Analysen versuchten dann erwartungsinduzierte emotionale Reaktionen zu messen. Dabei wurden in einem Analyseansatz emotionale Reaktionen auf Segmente mit hohem Informationsgehalt (die statistisch schwer vorhersagbar waren) mit denen mit niedrigem Informationsgehalt verglichen. In einem anderen Ansatz wurden die von den Teilnehmern als sehr unerwartet bewerteten Segmente mit den als sehr erwartet eingestuften Segmenten hinsichtlich ihrer emotionalen Wirkungen verglichen. Ergebnis beider Analysen war, dass nach Segmenten mit hohem Informationsgehalt und nach Segmenten, die als unerwartet beschrieben wurden, die subjektive und physiologische Erregung anstiegen. Damit konnte bestätigt werden, dass auch in einem im Konzert durchgeführten Experiment mit hoher ökologischer Validität das Verletzen von musikalischen Erwartungen zu emotionalen Reaktionen in unterschiedlichen Emotionskomponenten führen kann. 


\section{Zusammenfassung und Schlussbemerkung}

Mit Blick auf die soeben zitierten empirischen Arbeiten lässt sich zusammenfassend Folgendes feststellen: Es gibt experimentelle Belege für die Hypothese, dass einige musikinduzierte Emotionen durch Erwartungsprozesse bedingt sind. Dieser Zusammenhang wird vermutlich durch Mechanismen des auditiven statistischen Lernens vermittelt und konnte insbesondere für das Verletzen von melodischen Erwartungen nachgewiesen werden. Bisherige Forschungen konzentrierten sich bislang darauf, emotionale Reaktionen beim Verletzen von Erwartungen zu untersuchen, die sich durch die Bewertung musikalischer Ereignisse als unvorhergesagt und mit dem Entstehen von Überraschung erklären lassen. Somit besteht nur für einen Teil der von Huron im ITPRA-Modell vorgestellten emotionalen Reaktionen eine empirische Absicherung. Weitere Response-Typen, wie etwa die feine Differenzierung zwischen sprediction response`, sreaction response und sappraisal response`, erfordern weitere experimentelle Überprüfungen durch Versuchsbedingungen, die theoriegeleitet die unterschiedlichen Reaktionsarten abbilden und vergleichbar machen. Ebenfalls könnten die verschiedenen von Huron beschriebenen Erwartungsarten hinsichtlich ihrer Bedeutung für Emotionsentstehungsprozesse verglichen werden. Dazu ließen sich die Teilmodelle für dynamische und schematische Erwartungen innerhalb des IDYOM heranziehen.

Bislang ist auch wenig erforscht, inwieweit unterschiedliche performative Interpretationen ein und desselben Notentextes unterschiedliche Erwartungsqualitäten bedingen. Es ist anzunehmen, dass Interpreten durch die zeitliche, dynamische und klangfarbliche Gestaltung musikalischer Kompositionen bestimmte Hörererwartungen erzeugen können, die dann in der Folge erfüllt oder verletzt werden.

Die Beschäftigung mit erwartungsinduzierten Emotionen stellt somit auch weiterhin ein vielversprechendes Forschungsthema dar. Trotz einiger noch unbestätigter Vorhersagen zeigen sich erste Ergebnisse, die auch in größeren Kontexten Bedeutung erlangen könnten: Mit dem Nachweis der Tatsache, dass musikalische Strukturen aus sich selber heraus, ohne externe außermusikalische Referenz, emotionale Wirkung entfalten können, könnte ein wesentlicher Beitrag zum allgemeinen Verständnis von Musik und emotionalen Prozessen erreicht werden.

\section{Literatur}

Abdallah, Samer / Mark Plumbley (2002), »Information Dynamics: Patterns of Expectation and Surprise in the Perception of Music", Connection Science 23/2-3, 89-117.

Bharucha, Jamshed J. / Keiko Stoeckig (1986), »Reaction Time and Musical Expectancy: Priming of Chords", Journal of Experimental Psychology: Human Perception and Performance 12/4, 403-10.

Bharucha, Jamshed J. / Peter M. Todd (1989), »Modeling the Perception of Tonal Structure with Neural Nets«, Computer Music Journal 13/4, 44-53. 
Bigand, Emmanuel / Marion Pineau (1997), „Global Context Effects on Musical Expectancy", Perception Psychophysics 59/7, 1098-1107.

Bissell, Arthur Dart (1921), The Role of Expectation in Music, PhD. Dissertation, Yale University.

Blood, Anne J. / Robert J. Zatorre (2001), »Intensely Pleasurable Responses to Music Correlate with Activity in Brain Regions Implicated in Reward and Emotion«, Proceedings of the National Academy of Sciences of the United States of America 98/20, 11818-23.

Carrión, Ricardo E. / Benjamin Martin Bly (2008), »The Effects of Learning on Eventrelated Potential Correlates of Musical Expectancy«, Psychophysiology 45/5, 759-75.

Cochrane, Tom (2010), »Music, Emotions and the Influence of the Cognitive Sciences«, Philosophy Compass 11, 978-988.

Cuddy, Lola L. / Carole A. Lunney (1995), „Expectancies Generated by Melodic Intervals: Perceptual Judgments of Melodic Continuity«, Perception \& Psychophysics 57/6, 451-462.

Egermann, Hauke / Stephen McAdams (2013), »Empathy and Emotional Contagion as a Link between Recognized and Felt Emotions in Music Listening", Music Perception 31/2, 139-156.

Egermann, Hauke / Marcus Pearce / Geraint Wiggins / Stephen McAdams (2013), »Probabilistic Models of Expectation Violation Predict Psychophysiological Emotional Responses to Live Concert Music", Cognitive Affective Behavioral Neuroscience, 13/3, 533-553.

Egermann, Hauke / Mary Elizabeth Sutherland / Oliver Grewe / Frederik Nagel / Reinhard Kopiez / Eckart Altenmüller (2011), „Does Music Listening in a Social Context Alter Experience? A Physiological and Psychological Perspective on Emotion«, Musicae Scientiae 15/3, 307-323.

Fehr, Beverley / James A. Russell (1984), „Concept of emotion viewed from a prototype perspective«, Journal of Experimental Psychology: General, 113, 464-486.

Feldmann, Matthias (1998), Erwartungsdiskrepanz und emotionales Erleben von Musik, Hildesheim: Olms.

Huron, David (2006), Sweet Anticipation: Music and the Psychology of Expectation, Cambridge: MIT Press.

Janata, Peter (1995), „ERP Measures Assay the Degree of Expectancy Violation of Harmonic Contexts in Music", Journal of Cognitive Neuroscience 7/2, 153-164.

Juslin, Patrik N. / Daniel Västfjäll (2008), „Emotional Responses to Music: The Need to Consider Underlying Mechanisms", The Behavioral and Brain Sciences 31/5, 559-75, discussion 575-621.

Kivy, Peter (1990), Music Alone: Philosophical Reflections on the Purely Musical Experience, Ithaca, NY: Cornell University Press.

Koelsch, Stefan / Tom Fritz / Gottfried Schlaug (2008), „Amygdala Activity can be Modulated by Unexpected Chord Functions During Music Listening", Neuroreport 19/18, 1815-1819. 
Koelsch, Stefan / Simone Kilches / Nikolaus Steinbeis / Stefanie Schelinski (2008), »Effects of Unexpected Chords and of Performer's Expression on Brain Responses and Electrodermal Activity«, PloS one 3/7, e2631.

Konečni, Vladimir J. (2008), „Does Music Induce Emotion? A Theoretical and Methodological Analysis", Psychology of Aesthetics, Creativity and the Arts 2/2, 115-129.

Ladinig, Olivia / Henkjan Honing / Gábor Háden / István Winkler (2009), »Probing Attentive and Preattentive Emergent Meter in Adult Listeners without Extensive Music Training", Music Perception 26/4, 377-386.

LeDoux, Joseph (1996), The Emotional Brain, New York: Simon and Schuster.

Larson, Steve (2004), »Musical Forces and Melodic Expectations: Comparing Computer Models and Experimental Results«, Music Perception 21/4, 457-498.

Margulis, Elizabeth Hellmuth (2005), »A Model of Melodic Expectation«, Music Perception 22/4, 663-714.

Meyer, Leonard B. (1956), Emotion and Meaning in Music, Chicago: University of Chicago Press.

Narmour, Eugene (1990), The Analysis and Cognition of Basic Melodic Structures: The Implication-Realization Model, Chicago: Chicago University Press.

— (1991), »The Top-down and Bottom-up Systems of Musical Implication: Building on Meyer's Theory of Emotional Syntax«, Music Perception 9/1, 1-26.

_ (1992), The Analysis and Cognition of Melodic Complexity, Chicago: Chicago University Press.

Ockelford, Adam (2006), »Implication and Expectation in Music: A Zygonic Model«, Psychology of Music 34/1, 81-142.

Pearce, Marcus (2005), The Construction and Evaluation of Statistical Models of Melodic Structure in Music Perception and Composition, Diss., London, UK, Department of Computing, City University.

Pearce, Marcus / Geraint Wiggins (2004), »Improved Methods for Statistical Modelling of Monophonic Music", Journal of New Music Research 33/4, 367-385.

(2006), »Expectation in Melody: The Influence of Context and Learning«, Music Perception 23/5, 377-405.

Pearce, Marcus / María Herrojo Ruiz / Selina Kapasi / Geraint Wiggins / Joydeep Bhattacharya (2010), „Unsupervised Statistical Learning Underpins Computational, Behavioural and Neural Manifestations of Musical Expectation «, Neurolmage 50/1, 302-313.

Pearce, Marcus / Daniel Müllensiefen / Geraint Wiggins (2010), »The Role of Expectation and Probabilistic Learning in Auditory Boundary Perception: A Model Comparison«, Music Perception 39/10, 1365-1389.

Roseman, Ira J. / Ann Aliki Antoniou / Paul E. Jose (1996), „Appraisal Determinants of Emotions: Constructing a More Accurate and Comprehensive Theory«, Cognition \& Emotion 10/3, 241-278.

Salimpoor, Valorie N. / Mitchel Benovoy / Kevin Larcher / Alain Dagher / Robert J. Zatorre (2011), »Anatomically Distinct Dopamine Release During Anticipation and Experience of Peak Emotion to Music«, Nature Neuroscience 14, 257-262. 
Schellenberg, E. Glenn (1996), „Expectancy in Melody: Tests of the Implication-realization Model «, Cognition 58/1, 75-125.

Scherer, Klaus (1999), »Appraisal Theory«, in: Handbook of Cognition and Emotion, hg. von Tim Dalgleish und Mick J. Power, Chichester: Wiley, 637-663.

(2004), „Which Emotions Can be Induced by Music? What Are the Underlying Mechanisms? And How Can We Measure Them? ", Journal of New Music Research 33/3, 239-251.

(2005), „What Are Emotions? And How Can They Be Measured? «, Social Science Information 44/4, 695-729.

Schmuckler, Mark A. / Marilyn G. Boltz (1994), »Harmonic and Rhythmic Influences on Musical Expectancy«, Perception \& Psychophysics 56/3, 313-325.

Sloboda, John A. (1991), »Music Structure and Emotional Response: Some Empirical Findings", Psychology of Music 19/2, 110-120.

Sokolowski, Kurt (2002), „Emotion«, in: Allgemeine Psychologie, hg. von Jochen Müsseler und Wolgang Prinz, Heidelberg: Spektrum, 337-384.

Steinbeis, Nikolaus / Stefan Koelsch / John A. Sloboda (2006), »The Role of Harmonic Expectancy Violations in Musical Emotions: Evidence from Subjective, Physiological and Neural responses", Journal of Cognitive Neuroscience 18/8, 1380-1393.

Thompson, William Forde / Murray Stainton (1998), »Expectancy in Bohemian Folk Song Melodies, Evaluation of Implicative Principles for Implicative and Closural Intervals", Music Perception 15/3, 231-252.

Zanto, Theodore P. / Joel S. Snyder / Edward W. Large (2006), „Neural Correlates of Rhythmic Expectancy", Advances in Cognitive Psychology 2/2, 221-231.

Zillmann, Dolf (1971), »Excitation Transfer in Communication-Mediated Aggressive Behavior", Journal of Experimental Social Psychology 7, 419-434. 